\title{
Evolution de la teneur en césium 137 depuis 1967 dans l'eau de coco et le coprah en Polynésie française
}

\author{
L. KABIS de SAINT-CHAMAS *, J.P. BABLET*, C. ARNOULD**, \\ R. DUCOUSSO *
}

(Manuscrit reçu le 27 décembre 1989)

RÉSUMÉ La France procède à des expérimentations nucléaires en Polynésie française depuis 1966. D'abord aériennes, elles sont devenues souterraines depuis 1975. Dans le cadre de la surveillance de ces expérimentations, le contrôle de la teneur en césium 137 de la noix de coco est réalisé sur l'ensemble du territoire de la Polynésie française. Après avoir décrit la méthodologie, nous présentons les résultats des 2589 échantillons prélevées de 1967 à 1988. Les teneurs maximales trouvées depuis 1967 sont de $52 \mathrm{~Bq} \cdot \mathrm{kg}^{-1}$ pour l'eau de coco et de 289 Bq. $\mathbf{k g}^{-1}$ pour le coprah. La décroissance de la teneur en césium 137 est constante, sans discontinuité, à partir de 1974 quel que soit l'éloignement par rapport aux sites d'expérimentations. L'équivalent de dose d'irradiation engagé dû au césium 137, délivré par la consommation d'eau de coco et de coprah représente quelques microsieverts par an.

ABSTRACT Nuclear experiments have been carried out by France in french Polynesia since 1966. The tests were first atmospheric, they have been underground since 1975. The experiment monitoring programme includes monitoring of ${ }^{137} \mathrm{Cs}$ contents in coconuts from the whole french polynesian territory. The methodology is described and the results of the 2589 samples collected for 1967 to 1988 are presented. The maximum content found since 1967 is $52 \mathrm{Bg}^{\mathrm{kg}}{ }^{-1}$ for coconut water and $289 \mathrm{~Bq} \cdot \mathrm{kg}^{-1}$ for coconut copra. The decrease of ${ }^{137} \mathrm{Cs}$ content is constant without discontinuity, whatever the distance from the explosion sites. The committed dose equivalent from ${ }^{137} \mathrm{Cs}$ delivered by coconut water and copra consumption represents only a few microsieverts a year.

\section{INTRODUCTION}

Afin de maintenir le niveau de crédibilité de sa force nucléaire de dissuasion, la France a procédé sur les sites de Mururoa et de Fangataufa, entre 1966 et 1974, à des essais atmosphériques d'engins expérimentaux et d'armes. Depuis 1975, les expérimentations sont souterraines, en puits. Réalisés, au départ, à partir de la couronne émergée des atolls, les forages sont pratiqués en lagon depuis plusieurs années.

* Service mixte de contrôle biologique, BP 16, 91311 Montlhéry Cedex.

** Laboratoire d'étude et de surveillance de l'environnement, BP 519, Papeete, Polynésie française. 
Créé en 1964, le Service mixte de contrôle biologique (SMCB) a pour mission de contrôler la faune et la flore dans les zones intéressées par les essais nucléaires. Son effort porte notamment sur les denrées alimentaires. Parmi celles-ci, la noix de coco revêt une importance particulière.

L'eau de coco est largement consommée dans l'ensemble de la Polynésie et particulièrement sur atoll, où la ration journalière moyenne est estimée à 1 litre par personne. Le coprah, s'il entre pour une moindre part dans l'alimentation humaine directe, participe très largement à l'alimentation du bétail, directement sur atoll, ou après extraction de l'huile à Tahiti.

Il faut noter que la contamination par le césium 137 de la noix de coco a été considérée par les autorités américaines comme argument majeur dans la décision d'évacuer pour la seconde fois, en 1978, la population de l'atoll de Bikini [3].

Dans ce travail, nous présentons l'évolution de la teneur en césium 137 des noix de coco prélevées depuis 1967 par le SMCB et mesurées par le Laboratoire d'études et de surveillance de l'environnement (LESE). L'étude porte à la fois sur l'évolution dans le temps et sur la répartition géographique des radionucléides présents dans l'eau de coco et dans le coprah.

\section{MATÉRIELS ET MÉTHODES}

Les programmes de prélèvements du SMCB répondent à un double souci :

- pouvoir dresser à tout moment la carte radioécologique de la Polynésie française;

- s'assurer que l'alimentation ne risque pas d'approcher les limites annuelles d'incorporation recommandées par la Commission internationale de protection radiologique (CIPR) et adoptées par la France [4], en ciblant notamment les îles les plus peuplées.

C'est pourquoi le SMCB contrôle 43 îles ou atolls, répartis dans la totalité des archipels de la Polynésie française, et, que parmi eux, on trouve les îles ou atolls les plus peuplés. On peut estimer que l'alimentation de $90 \%$ de la population est constamment et directement surveillée.

Pour simplifier la présentation des résultats de ce contrôle, l'ensemble de la Polynésie française a été divisé en quatre zones tenant compte de l'éloignement de la source, l'atoll de Mururoa constituant la première zone. Le détail des îles ou atolls des trois autres est donné par le tableau I. 
TENEUR EN CÉSIUM 137 DANS L'EAU DE COCO ET DE COPRAH EN POLYNÉSIE

TABLEAU I

Lieux de prélèvements des noix de coco et répartition des échantillons analysés

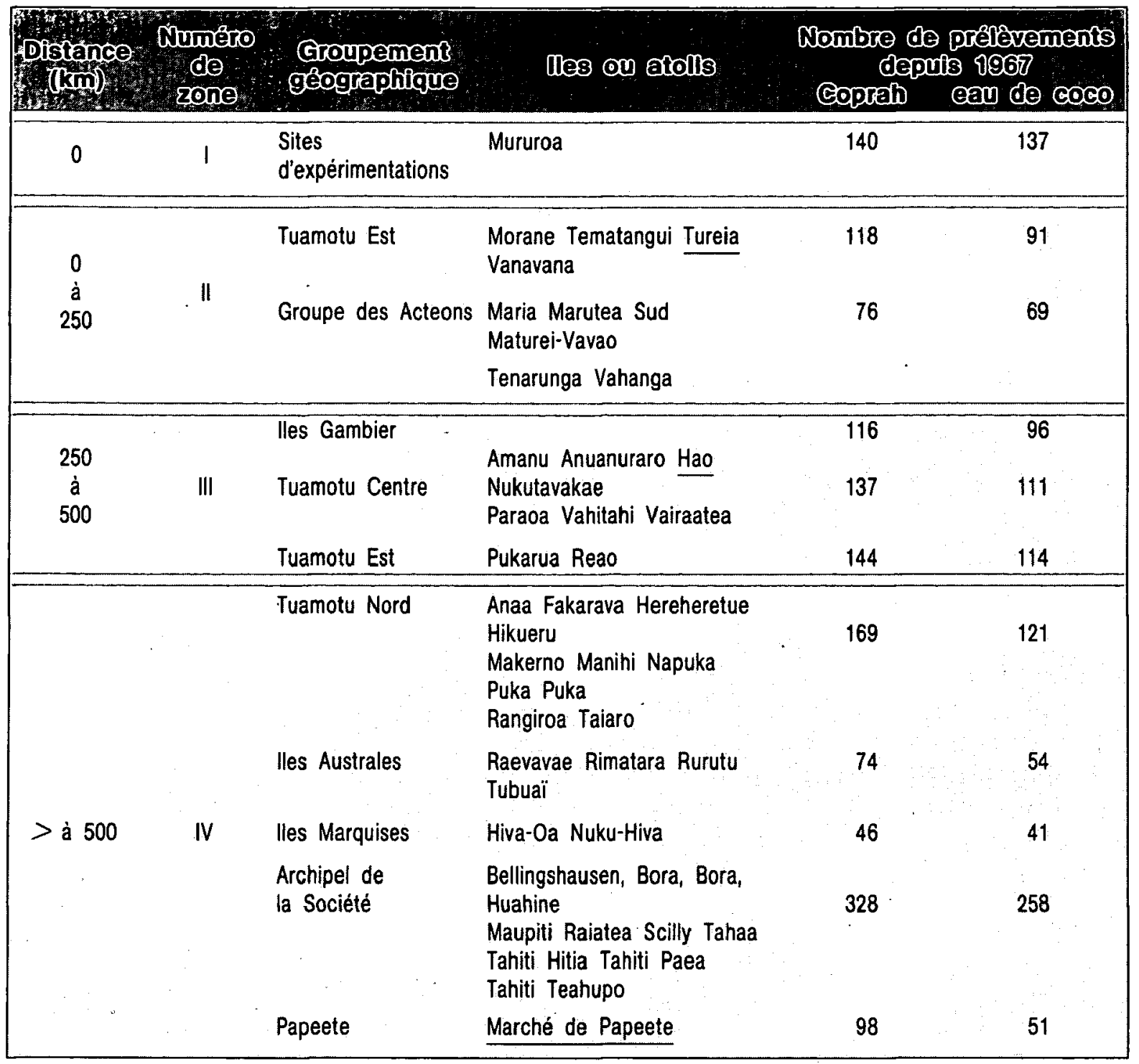

\subsection{Echantillons}

La constitution des échantillons soumis aux mesures a nécessité le prélèvement d'environ 70000 noix de coco entre 1967 et 1988 inclus. Selon que l'analyse ait porté sur l'eau de coco ou sur le coprah, les noix sont prélevées à des stades de maturation différents.

\subsubsection{Eau de coco}

Dans le but d'analyser l'eau de coco, les noix sont prélevées jeunes, avec l'écorce encore verte. A ce stade, elles répondent à l'appellation via via en langage paumotu [7]. C'est à ce moment que l'eau est la plus abondante dans la noix, et qu'elle est la plus agréable à boire. Pour obtenir une sensibilité convenable, il faut mesurer $50 \mathrm{~g}$ de cendres. Le taux de cendres de l'eau de coco étant de l'ordre de 0,5\% [9], il faut recueillir 10 litres d'eau, ce qui correspond au contenu de 30 à 40 noix. 


\subsubsection{Coprah}

Pour l'analyse du coprah les noix sont prélevées à maturité. A ce stade, leur écorce est brun clair et elle correspond au stade opaa [1]. Le taux de cendres du coprah étant un peu supérieur à $1 \%$ [9], il faut recueillir au moins $4 \mathrm{~kg}$ de coprah pour constituer un échantillon de $50 \mathrm{~g}$. Ceci correspond à environ 20 noix.

\subsection{Traitement préalable à la mesure}

Les échantillons sont pesés, puis desséchés à l'étuve à $120^{\circ} \mathrm{C}$ jusqu'à poids constant. L'opération dure environ $48 \mathrm{~h}$. Le taux d'humidité est en moyenne de $95 \%$ pour l'eau de coco et de $45 \%$ pour le coprah [9].

Après dessiccation, les échantillons sont incinérés pendant $24 \mathrm{~h}$ à $500^{\circ} \mathrm{C}$, sans programmation du temps de montée en température. Les cendres sont pesées puis homogénéisées avant prélèvement de $50 \mathrm{~g}$ qui suffisent à remplir des boîtes cylindriques géométriquement identiques, de $60 \mathrm{~mm}$ de diamètre pour $30 \mathrm{~mm}$ de hauteur.

\subsection{Mesure}

La mesure de césium 137 s'effectue par spectrométrie du rayonnement $\gamma$ émis directement par l'échantillon défini ci-dessus. Malgré les progrès réalisés par l'instrumentation de métrologie nucléaire, notamment la mise sur le marché de détecteurs de rayonnement $y$ à jonction de germanium-lithium au milieu des années 70 , puis de germanium hyperpur au début des années 80 , nos échantillons ont tous été mesurés par des détecteurs à cristal d'iodure de sodium activité au thallium ( $\mathrm{Nal} \mathrm{Tl}$ ), afin d'éviter d'introduire une variation dans les paramètres au cours du temps.

Ces détecteurs Nal Tl (Quartz et Silice) sont de dimension $4 \times 3$ ". Les analyseurs d'amplitude associés ont été de type SA 40, puis IN 96 (Intertechnique). Le temps d'acquisition est fixé à $200 \mathrm{~min}$, l'échantillon étant protégé du rayonnement extérieur par une enceinte blindée CBMJ (Lemer) munie d'un passeur automatique. Les spectres, stockés à l'origine sur ruban perforé puis sur disques souples, sont dépouillés à l'aide d'un logiciel semiautomatique. Les résultats correspondant à chacun des échantillons sont exprimés en becquerels par kilogramme de produit frais, ramenés au jour du prélèvement. lls sont conservés dans une banque de données informatique exploitée par un mini-ordinateur VAX 8250 (DEC).

A partir de 1981, les résultats obtenus par le LESE font l'objet d'intercomparaisons avec le Service central de protection contre les rayonnements ionisants (SCPRI), laboratoire de référence de l'Organisation mondiale de la santé.

\section{RÉSULTATS}

Le tableau Il donne les moyennes annuelles de la teneur en césium 137 de l'eau de coco et du coprah dans les quatre zones précédemment définies. 
C'est dans la zone II que l'on trouve les moyennes les plus élevées. Pour l'eau de coco, c'est en 1974 que la moyenne $\left(11,44 \mathrm{~Bq} \cdot \mathrm{kg}^{-1}\right)$ est relevée, un prélèvement du mois d'avril sur l'atoll Maria des Actéons ayant une teneur en ${ }^{137} \mathrm{Cs}$ de $52 \mathrm{~Bq} \cdot \mathrm{kg}^{-1}$. C'est la valeur maximale trouvée sur un échantillon d'eau de coco dans l'ensemble de la Polynésie française depuis 1967. Pour le coprah, la moyenne est relevée en 1971 par un prélèvement effectué également sur l'atoll Maria des Actéons ayant une teneur en ${ }^{137} \mathrm{Cs}$ de $289 \mathrm{~Bq} \cdot \mathrm{kg}^{-1}$ (valeur maximale trouvée sur un échantillon de coprah depuis 1967).

TABLEAU II

Moyennes annuelles de la teneur en césium 137 (en Bq.kg ${ }^{-1}$ frais)

\begin{tabular}{|c|c|c|c|c|c|c|c|c|}
\hline t. & $\begin{array}{l}\text { Eao } \\
\text { coco } \\
\text { coco }\end{array}$ & Goprah & $\begin{array}{l}20 \\
\text { Eav de } \\
\text { Coco }\end{array}$ & Goprah & $\begin{array}{l}\frac{20}{20} \\
\text { Eav de } \\
\text { Coco }\end{array}$ & Goprah & $\begin{array}{l}\text { Zo } \\
\text { Eav de } \\
\text { coco. }\end{array}$ & av \\
\hline 1967 & 6,18 & 5,49 & 6,46 & 20,44 & 6,75 & 18,81 & 5,12 & 7,32 \\
\hline 1968 & 4,99 & 11,22 & 3,81 & 14,21 & 1,05 & 7,19 & 3,53 & 9,35 \\
\hline 1969 & 2,49 & 8,07 & 3,59 & 15,98 & 2,16 & 9,95 & 2,13 & 7,79 \\
\hline 1970 & 2,24 & 7,84 & 9,79 & 31,36 & 1,88 & 10,08 & 1,58 & 5,41 \\
\hline 1971 & 3,61 & 6,66 & 6,99 & 51,74 & 2,21 & 9,15 & 1,39 & 4,32 \\
\hline 1972 & 2,26 & 7,68 & 8,62 & 25,36 & 2,08 & 8,95 & 1,22 & 4,58 \\
\hline 1973 & 1,29 & 7,84 & 9,93 & 22,81 & 2,77 & 10,66 & 0,81 & 5,16 \\
\hline 1974 & 0,94 & 5,27 & 11,44 & 41,06 & 2,69 & 12,85 & 1,49 & 4,63 \\
\hline 1975 & 0,85 & 4,37 & 3,14 & 12,48 & 2,58 & 7,54 & 0,74 & 2,62 \\
\hline 1976 & 0,76 & 6,10 & 3,87 & 28,58 & 2,20 & 10,12 & 0,51 & 2,89 \\
\hline 1977 & 0,65 & 4,70 & 6,28 & 29,83 & 2,88 & 11,27 & 0,60 & 3,11 \\
\hline 1978 & 1,20 & 4,74 & 7,62 & 17,62 & 1,78 & 4,55 & 0,81 & 5,68 \\
\hline 1979 & 0,82 & 3,44 & 2,61 & 20,83 & 0,54 & 2,13 & 0,80 & 2,72 \\
\hline 1980 & 0,65 & 1,87 & 3,11 & 20,28 & 0,77 & 2,67 & 0,35 & 3,19 \\
\hline 1981 & 0,18 & 3,48 & 4,13 & 19,29 & 1,03 & 4,15 & 0,68 & 3,07 \\
\hline 1982 & 0,61 & 1,42 & 4,88 & 8,57 & 0,56 & 4,35 & 1,07 & 2,60 \\
\hline 1983 & 0,36 & 2,61 & & 10,91 & 0,44 & 4,85 & 1,46 & 2,56 \\
\hline 1984 & & 1,04 & 2,66 & 11,93 & 0,47 & 6,88 & 0,58 & 2,70 \\
\hline 1985 & 0,20 & 0,99 & 2,39 & 6,85 & 0,56 & 2,49 & 0,85 & 1,48 \\
\hline 1986 & 0,32 & 1,48 & 1,07 & 5,49 & 0,23 & 0,61 & 0,68 & 2,30 \\
\hline 1987 & 0,44 & 0,96 & 2,05 & 10,19 & 0,66 & 1,31 & 0,48 & 5,89 \\
\hline 1988 & 0,92 & 3,24 & 1,24 & 4,99 & 0,62 & 3,71 & 0,55 & 2,34 \\
\hline
\end{tabular}


Les figures 1 à 4 visualisent en coordonnées semi-logarithmiques l'évolution dans le temps de la teneur en césium 137 de l'eau de coco pour chaque zone, ainsi que pour le lieu le plus peuplé de la zone.

Les noix de coco de l'atoll de Mururoa ont pratiquement toujours été prélevées à deux endroits bien précis, ce qui explique une certaine homogénéité des résultats comme le montre la figure 1.

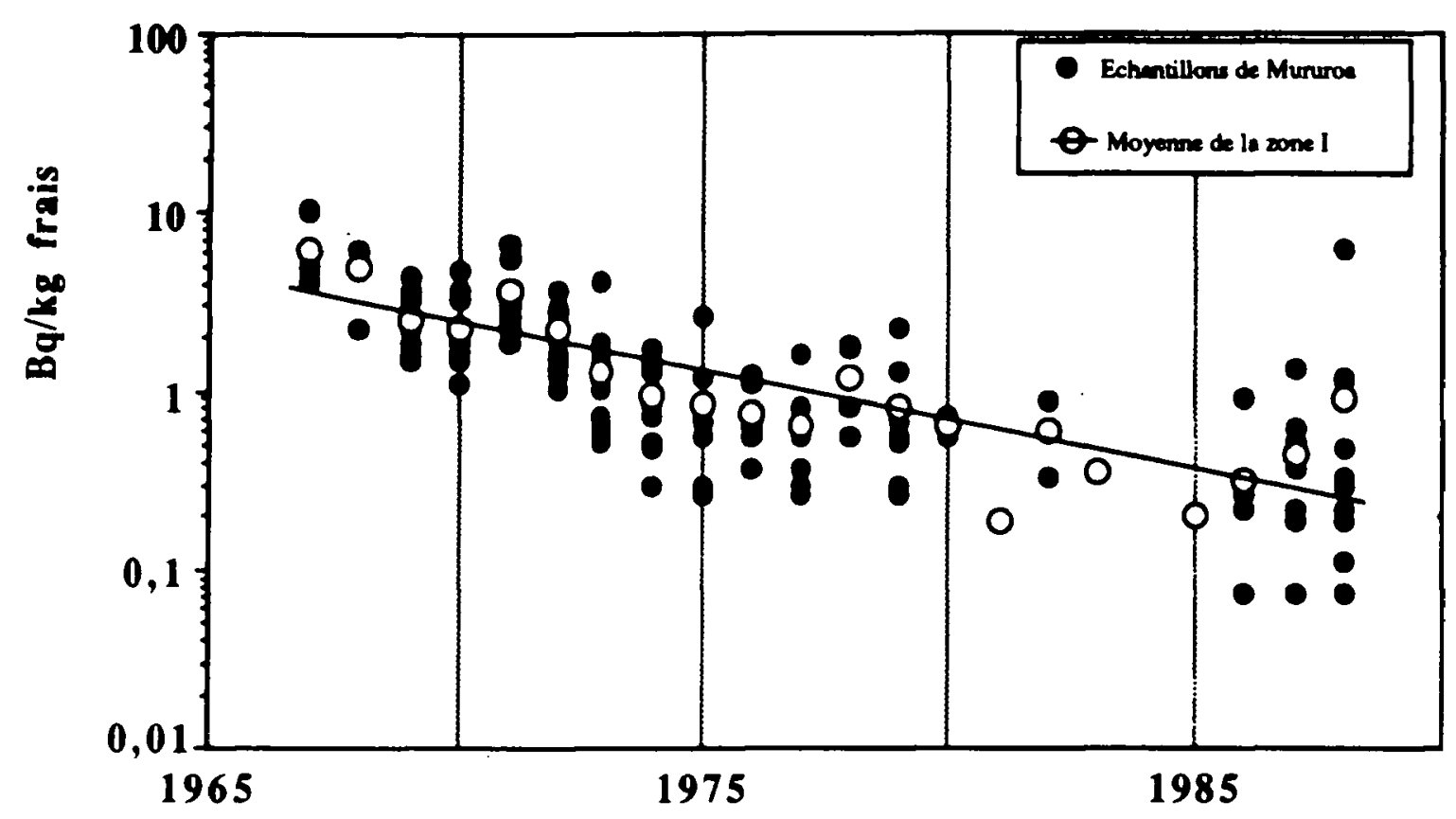

Fig. 1. - Teneur en cósium 137 de l'eau de coco a Mururoe.

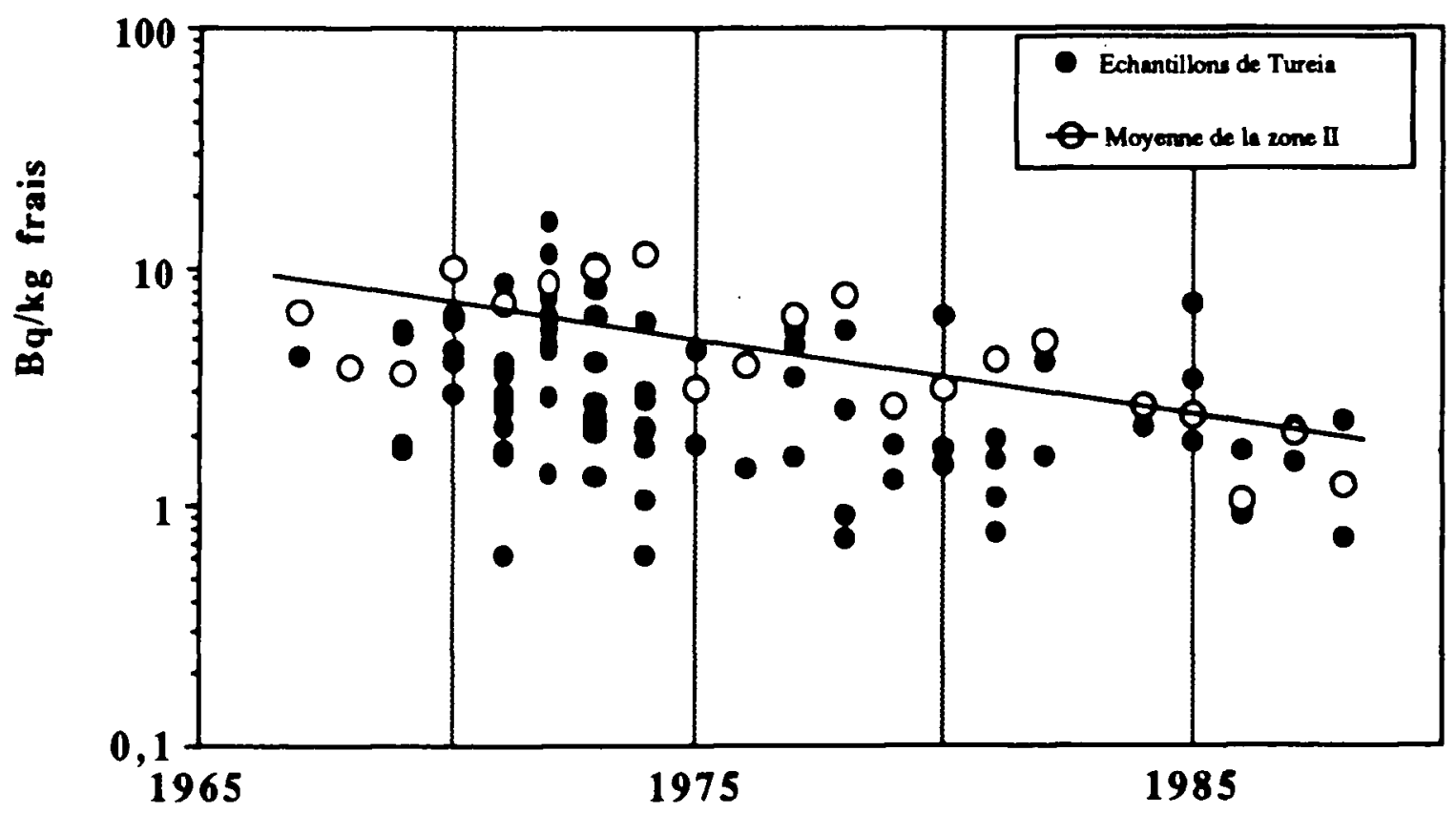

Fig. 2. - Teneur en césium 137 de l'eau de coco a Turela et moyenne de la zone II. 
La figure 2 donne la teneur en césium 137 des différents échantillons prélevés à Tureia, (deux échantillons supérieurs à $10 \mathrm{~Bq} . \mathrm{kg}^{-1}$ en 1972) ainsi que la moyenne de la zone II.

Dans la zone III et à Hao, atoll le plus peuplé de cette zone, la teneur en césium 137 a toujours été inférieure à $10 \mathrm{~Bq} \cdot \mathrm{kg}^{-1}$ et diminue depuis 1967 (fig. 3).

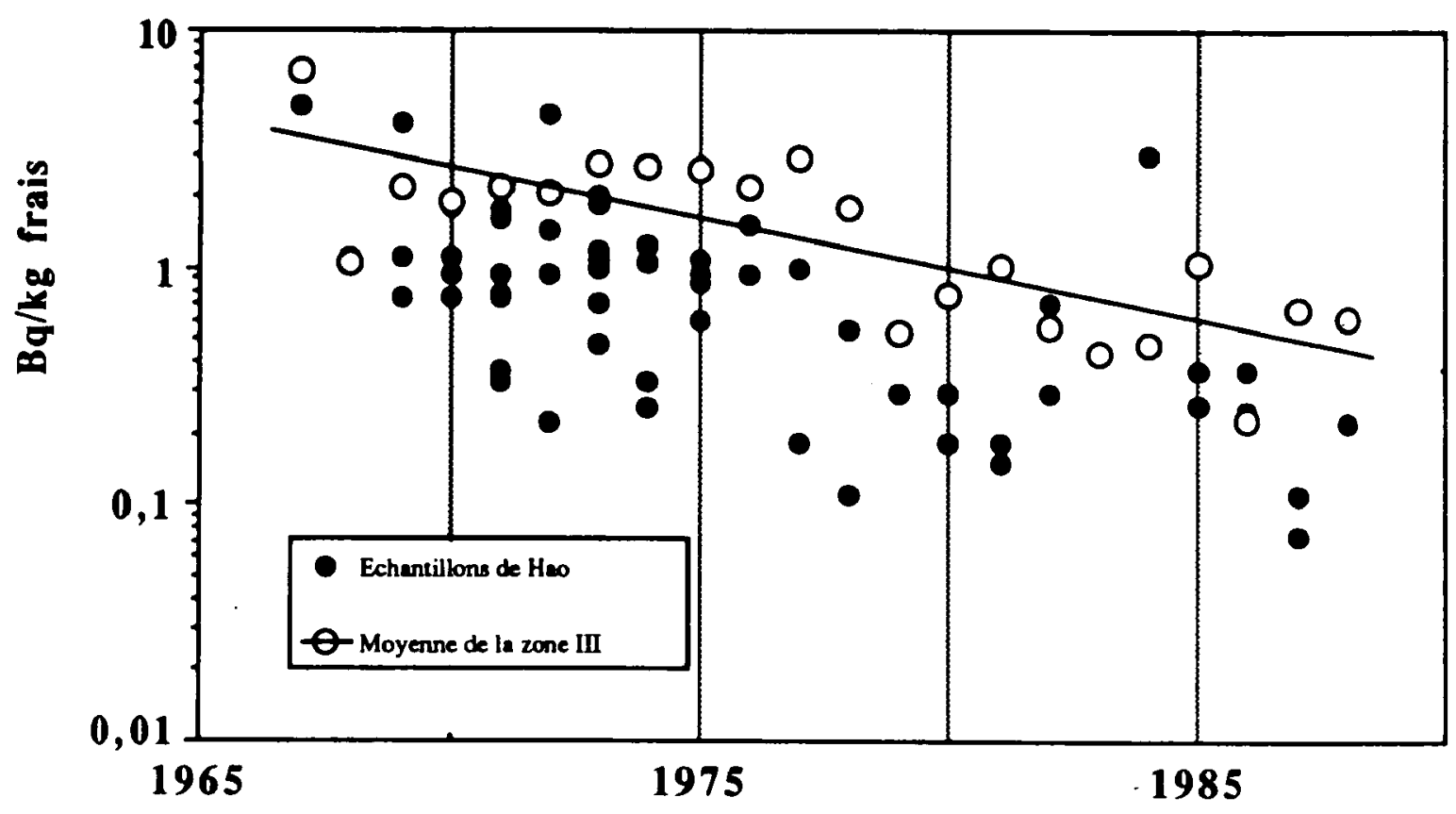

Flg. 3. - Teneur en céslum 137 de l'eau de coco a HaO et moyenne de la zone III.

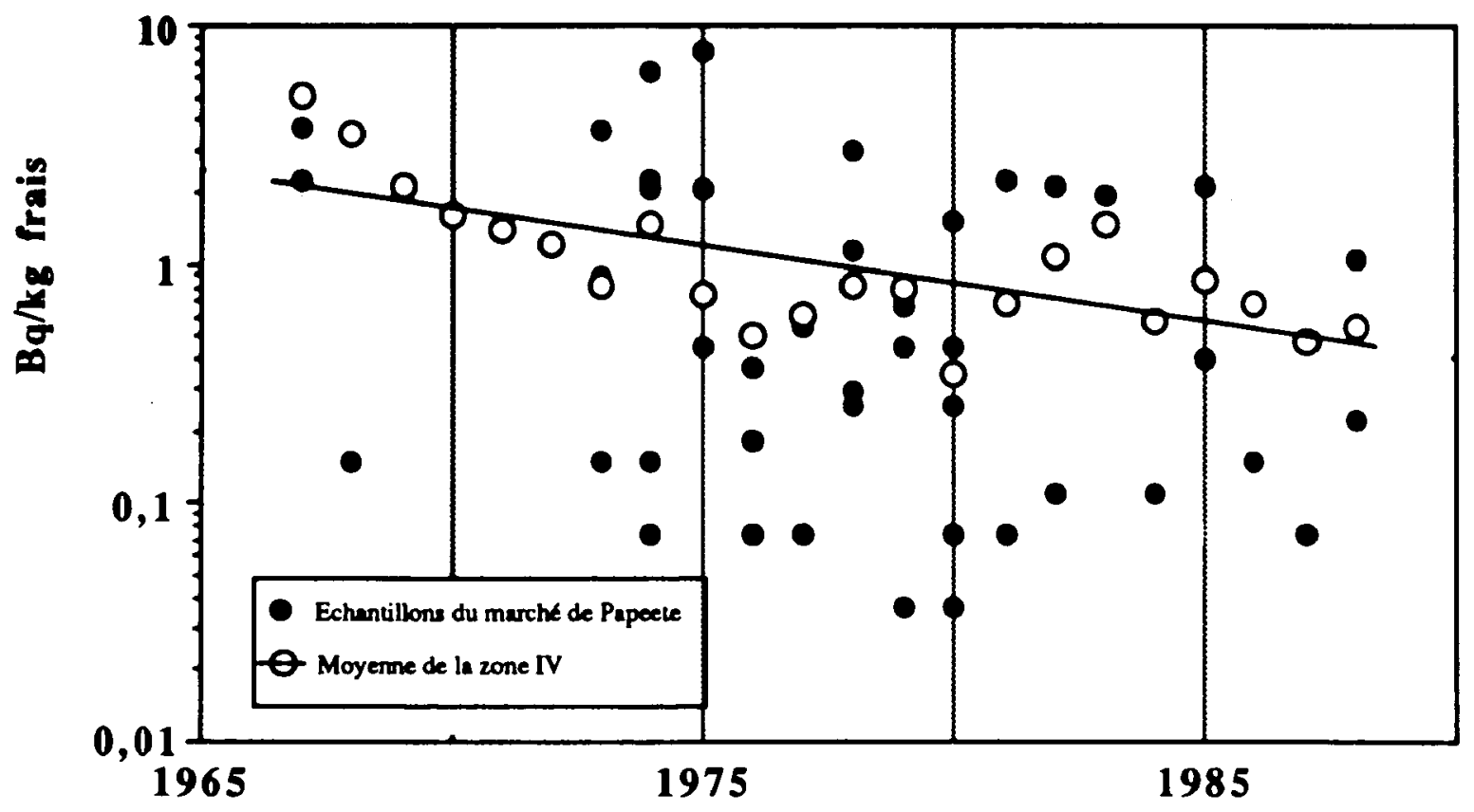

Flg. 4. - Teneur en céslum 137 de l'eau de coco au Marché do Papeote et moyenne de la zone IV. 
La figure 4 montre une grande dispersion dans les résultats pour. les échantillons du marché de Papeete. Ceci est dû au fait que ce marché est approvisionné par des noix de coco provenant non seulement des différentes communes de lîle de Tahiti, mais aussi des îles avoisinantes de l'archipel de la Société, voire d'îles ou d'atolls plus éloignés avec le développement des transports interinsulaires. Aucune teneur supérieure à $10 \mathrm{~Bq} \cdot \mathrm{kg}^{-1}$ n'a été observée depuis 1967.

Les figures 5 à 8 montrent, pour le coprah, la même évolution que pour l'eau de coco, dans un mode de présentation identique.

Comme pour l'eau de coco, on observe une certaine homogénéité dans les résultats. Seuls quelques échantillons ont une teneur en césium 137 supérieure à 10 Bq. $\mathrm{kg}^{-1}$ (fig. 5).

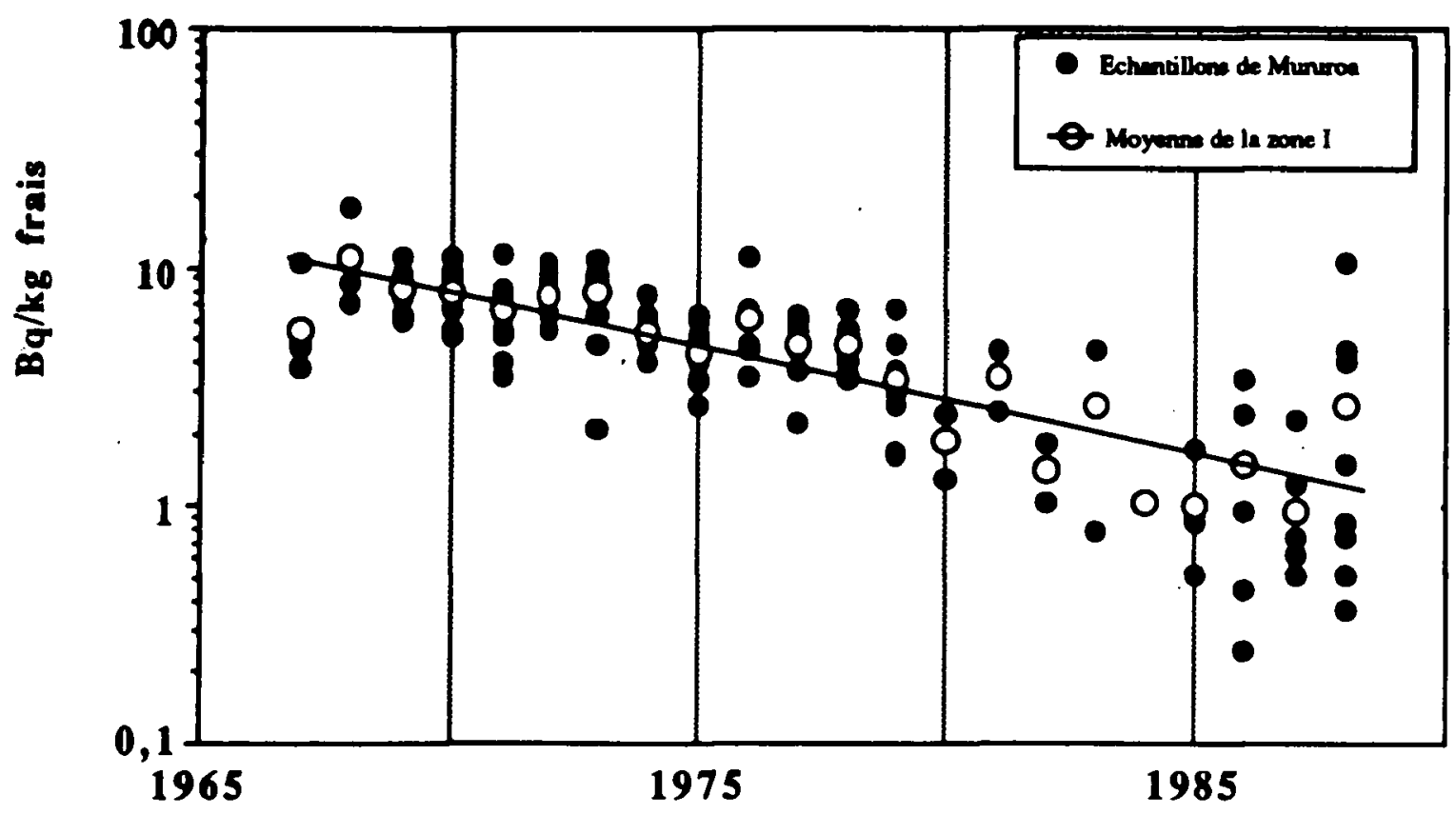

Fig. 5. - Teneur en céslum 137 du coprah d Mururoe.

Sur l'atoll de Tureia, les teneurs en césium 137 sont maiximales en 1970, puis diminuent pour se situer autour de $10 \mathrm{~Bq} \cdot \mathrm{kg}^{-1}$ en 1988 (fig. 6).

La figure 7 montre que la teneur en césium 137 des échantillons de Hao n'a jamais dépassé 20 Bq. .g $^{-1}$. 
TENEUR EN CÉSIUM 137 DANS L'EAU DE COCO ET DE COPRAH EN POLYNÉSIE

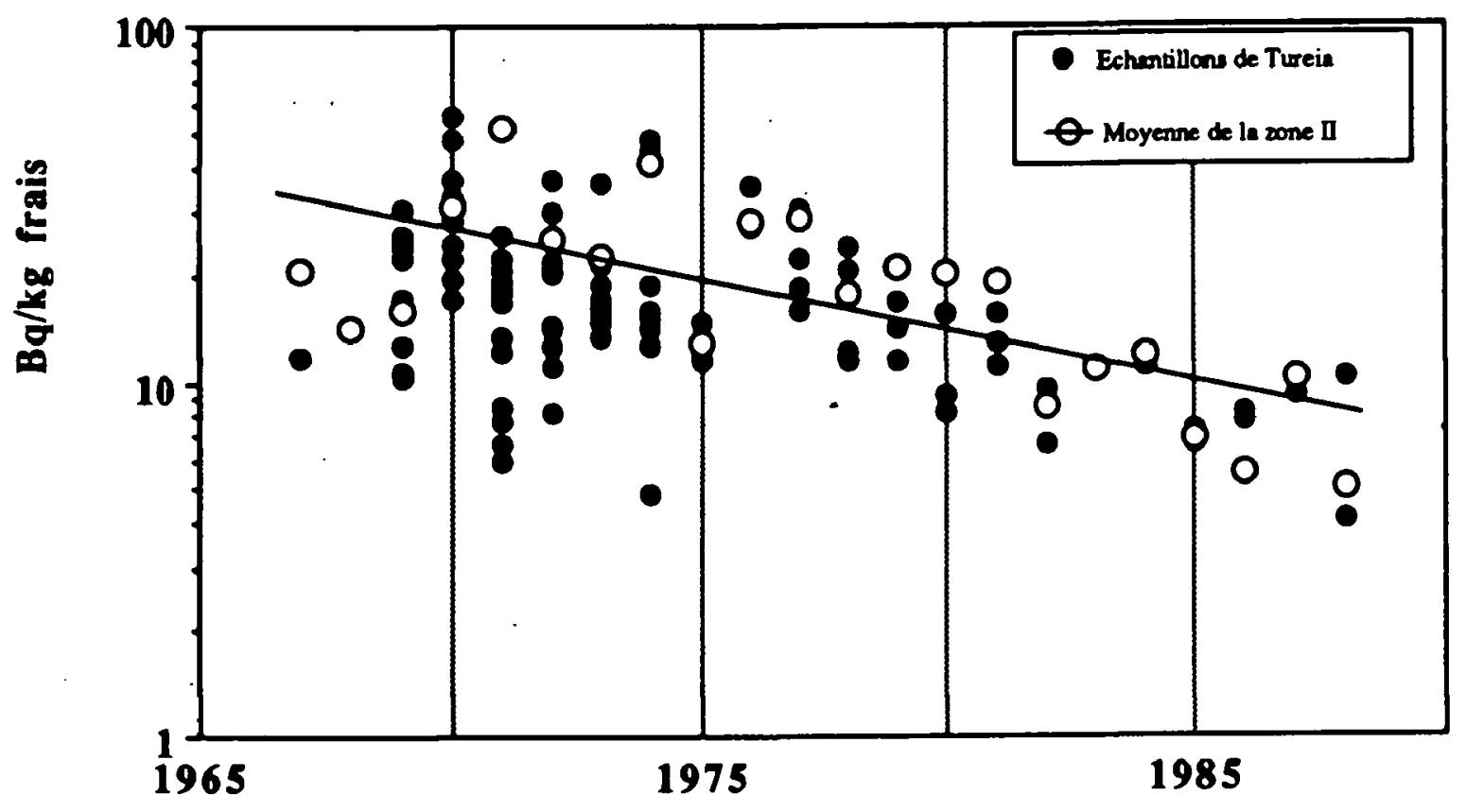

Fig. 6. - Tenour en césium 137 du coprah a Turala et moyenne de la zone ll.

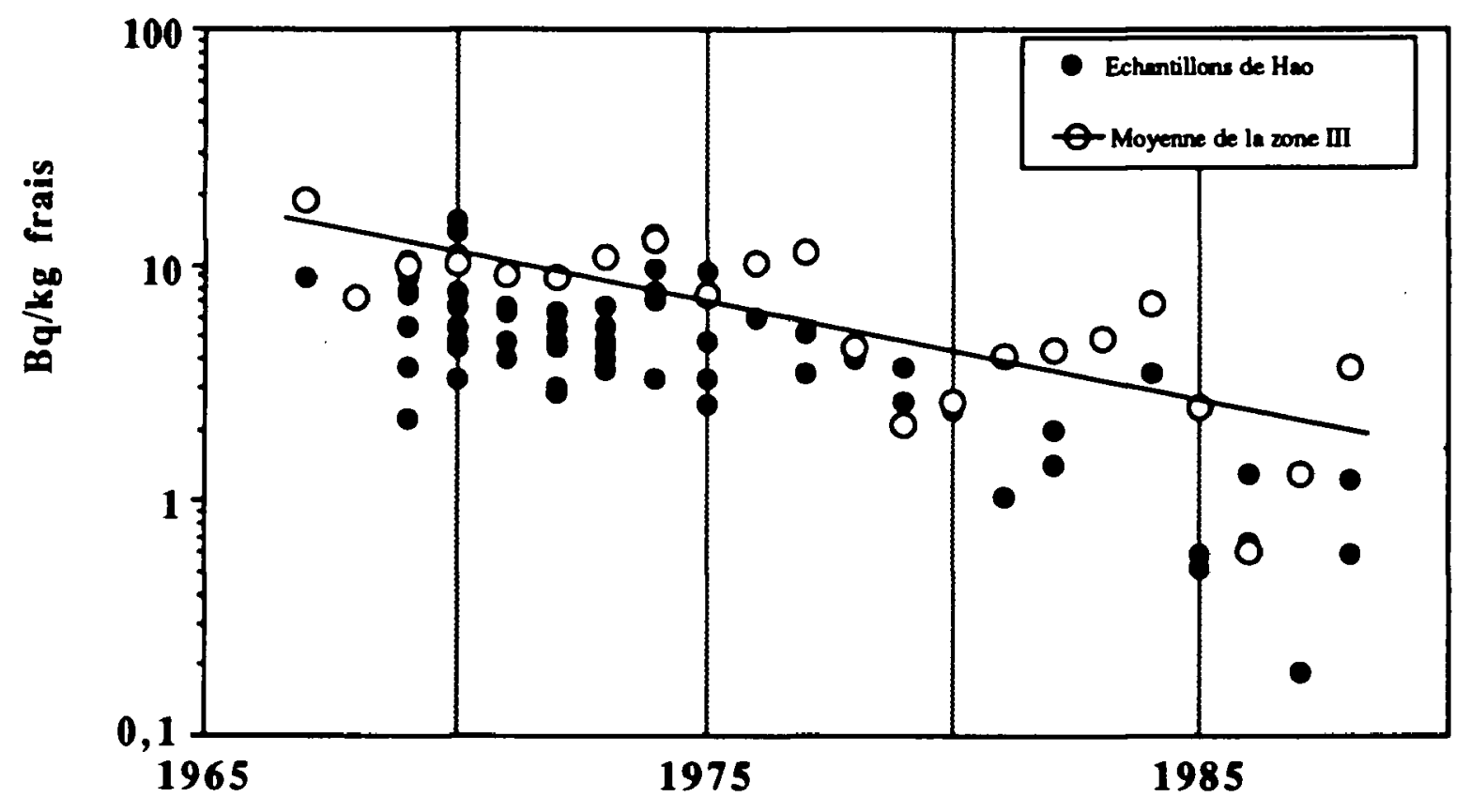

Fig. 7. - Teneur en céslum 137 du coprah \&aO et moyenne de la zone III. 
Ici aussi, on trouve une grande dispersion des teneurs en césium 137 des noix de coco du marché de Papeete (fig. 8). Depuis 1967, aucun prélèvement n'a dépassé des teneurs supérieures à 10 Bq. $\mathrm{kg}^{-1}$.

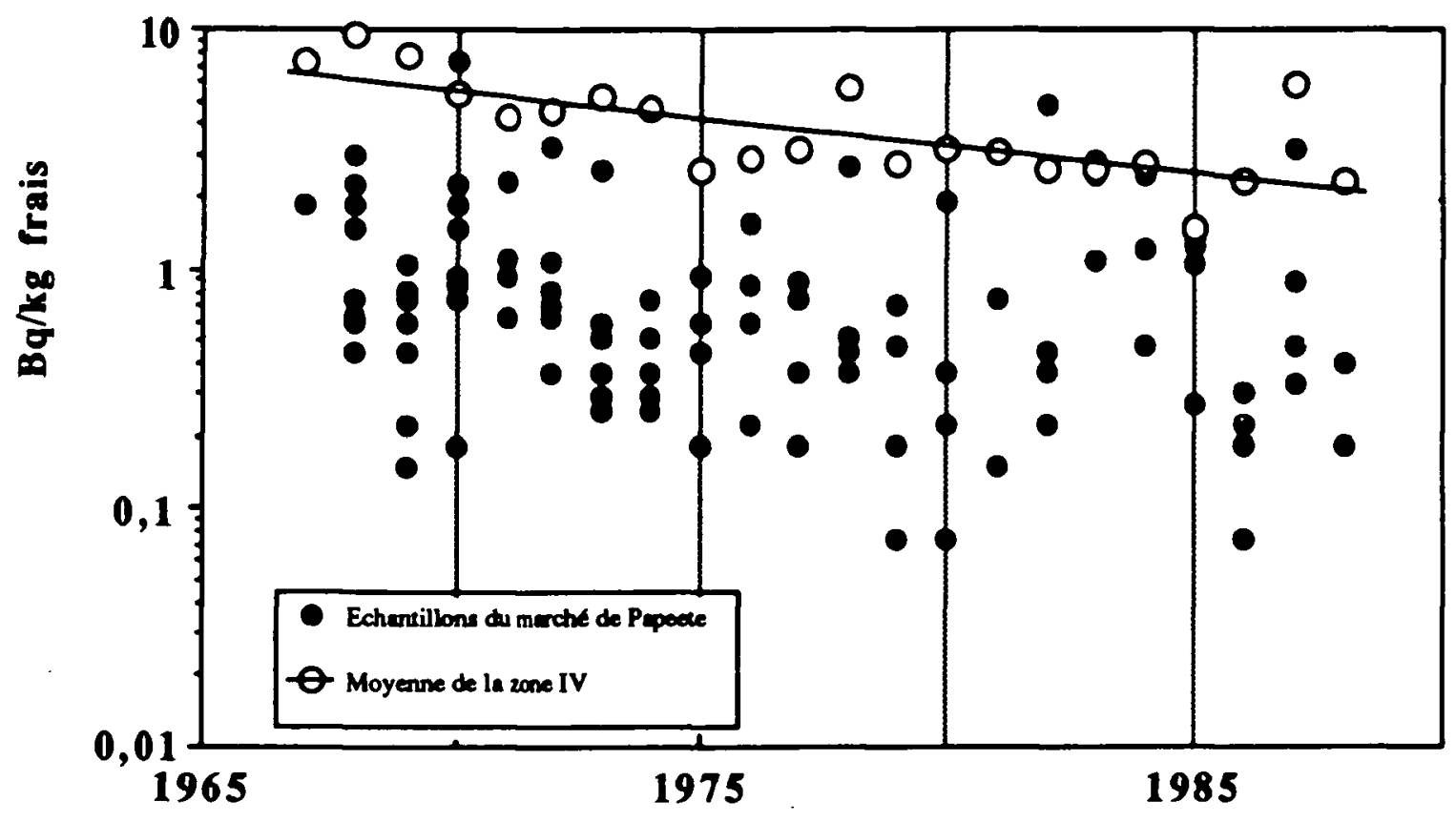

Fig. 8. - Tenour en césfum 137 du coprah au marché de Papoete et moyenne de la zone IV.

\section{DISCUSSION}

Pour les deux séries d'échantillons, la décroissance de la teneur en césium 137 est constatée pratiquement depuis le début de la période d'observation et notamment sans discontinuité à partir de 1974 , dernière année des essais atmosphériques. II est, par conséquent, manifeste qu'aucune injection de ce radionucléide ne s'est produite dans l'environnement polynésien depuis le début des expérimentations souterraines. De plus, l'absence de grandes variations pendant la période allant de 1967 à 1974 signifie que les retombées consécutives aux essais aériens de Mururoa et de Fangataufa n'ont présenté qu'une partie des retombées mondiales. Ceci est confirmé par l'évaluation à $16 \%$ de cette contribution française aux retombées mondiales dans l'hémisphère austral, faite par Moroney en 1979 [10]. Ceci est également confirmé par les résultats de l'étude menée sur la répartition, dans le temps et dans l'espace, d'un autre radionucléide, le strontium 90 , mesuré dans les dents des humains en Polynésie française [2].

Enfin, nos résultats sont en accord avec ceux concernant l'Argentine [12] qui montrent que la concentration du césium 137 dans la ration alimentaire est maximale, dans ce pays de l'hémisphère sud, entre 1964 et 1966 (environ 7,5 Bq. $\mathrm{kg}^{-1}$ ) et ne fait ensuite que décroître. 
Pour ce qui concerne la répartition géographique, on pouvait s'attendre à observer un gradient de décroissance entre le terme source que constituent les lieux d'expérimentation et les zones les plus éloignées. Deux constatations viennent à l'encontre de ce raisonnement:

- les teneurs en césium 137 sont moins élevées que celles que l'on pouvait attendre à Mururoa, elles y sont même moins élevées que dans la zone II et notamment à Tureia et Reao ;

- elles sont plus élevées que ne le voudrait l'éloignement dans des prélèvements effectués au marché de Papeete.

“L'anomalie" trouvée à Mururoa s'explique par la technologie des essais atmosphériques français. Très tôt après les débuts des expérimentations du Pacifique, les engins expérimentaux ont été placés à grande hauteur sous ballon captif. La boule de feu consécutive à l'explosion prenait très vite de l'altitude, entraînant avec elle les produits de fission, dont le césium 137. Ceci explique la faible contamination des atolls et, par voie de conséquence, la faible contamination de sa faune et de sa flore. C'est pourquoi le site pouvait être réoccupé immédiatement après chaque essai. Les conditions météorologiques retenues pour autoriser un tir comportaient notamment une direction de vent d'ouest-sud-ouest vers l'est-nord-est. Les atolls de Tureia et de Reao, situés en marge de cet axe ont parfois été soumis à la frange des retombées dites "locales". La contamination des noix de coco, encore qu'elle soit restée faible, y est relativement plus élevée qu'ailleurs, et reflète ces conditions expérimentales.

"L'anomalie" inverse rencontrée dans les prélèvements provenant du marché de Papeete s'explique par la diversité d'origine des noix vendues. Certaines peuvent provenir de la zone II, rehaussant la moyenne d'autant.

\section{TABLEAU III}

Equivalent de dose efficace annuel dû au césium 137 chez l'adulte. Comparaison entre l'ensemble de la ration alimentaire et l'ingestion d'eau de coco et de coprah pour les années 1971 et 1988 .

\begin{tabular}{|c|c|c|c|c|c|c|}
\hline \multirow{2}{*}{ 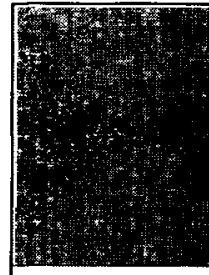 } & \multicolumn{4}{|c|}{ 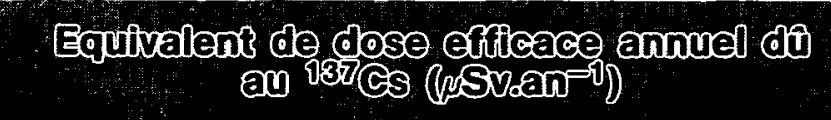 } & \multirow{2}{*}{\multicolumn{2}{|c|}{$\begin{array}{l}\text { Paru de Peau de coco } \\
\text { et du coprah dans } \\
\text { le fotal en ob }\end{array}$}} \\
\hline & $\begin{array}{l}\text { Total } \\
1977\end{array}$ & $\begin{array}{l}\text { jestion } \\
9988\end{array}$ & $\begin{array}{c}\text { Par Pe } \\
\text { ê } 0 \\
1970\end{array}$ & $\begin{array}{l}\text { goco } \\
\text { wah } \\
\text { 1988 }\end{array}$ & & \\
\hline Tureia & 55,10 & 21,96 & 35,00 & 15,52 & 64 & 71 \\
\hline Hao & 4,44 & 0,83 & 3,55 & 0,58 & 80 & 70 \\
\hline Papeete & 2,21 & 1,74 & 0,19 & 0,13 & 9 & 7 \\
\hline
\end{tabular}

* Données de 1973. 
L'interprétation sanitaire peut se faire en évaluant la dose d'exposition interne due au césium 137, délivrée par la consommation d'eau de coco et de coprah, en chacun des lieux retenus comme étant le plus peuplé de chaque zone en dehors de Mururoa. Cette évaluation tient compte des résultats de mesures de radioactivité et des quantités supposées être consommées en chaque lieu [6]. Ce calcul est présenté dans le tableau III pour deux années de référence : 1971 année où la contamination a été maximale, 1988 dernière année où les résultats complets sont disponibles. L'équivalent de dose efficace annuel dû à la présence de césium 137 dans les noix de coco exprimé en microsievert, est comparé à l'équivalent de dose efficace annuel dû à la présence de césium 137 dans l'ensemble de la ration alimentaire. La proportion du premier par rapport au second est présentée en pourcentage. Le tableau IV présente le même calcul appliqué au régime alimentaire des enfants de 5 ans (le tableau III s'applique au régime alimentaire des adultes).

Les données utilisées se rapportent aux années 1971 et 1988 (sauf pour Papeete où, par manque de données en 1971, nous avons pris celles de 1973 et 1988) communiquées comme tous les ans au Comité scientifique des nations-unies pour l'étude des rayonnements ionisants (United nations scientific committee on the effect of atomic radiations UNSCEAR). Toutefois, afin de pouvoir comparer, pour toutes les séries de valeurs de césium 137, nous avons utilisé les régimes alimentaires retenus actuellement [11].

TABLEAU IV

Equivalent de dose efficace annuel dû au césium 137 chez l'enfant de 5 ans d'âge.

Comparaison entre l'ensemble de la ration alimentaire et l'ingestion d'eau de coco et de coprah pour les années 1971 et 1988 .

\begin{tabular}{|c|c|c|c|c|c|c|}
\hline & \multicolumn{4}{|c|}{$\begin{array}{c}\text { Equivalent de dose efficace annuel dû } \\
\text { au }{ }^{137} \mathrm{Cs}\left(\mu \mathrm{Sv} \cdot \mathrm{an}^{-1}\right)\end{array}$} & \multirow{2}{*}{\multicolumn{2}{|c|}{$\begin{array}{l}\text { Part de l'eau de coco } \\
\text { et du coprah dans } \\
\text { le total en } \%\end{array}$}} \\
\hline & \multicolumn{2}{|c|}{ Total par ingestion } & \multicolumn{2}{|c|}{$\begin{array}{l}\text { Par l'eau de coco } \\
\text { et le coprah }\end{array}$} & & \\
\hline & 1971 & 1988 & 1971 & 1988 & 1971 & 1988 \\
\hline Tureia & 15,58 & 5,80 & 7,50 & 3,06 & 48 & 53 \\
\hline Hao & 1,46 & 0,23 & 1,12 & 0,17 & .77 & 74 \\
\hline Papeete & $1,01^{*}$ & 1,06 & $0,09^{*}$ & 0,06 & 9 & 6 \\
\hline
\end{tabular}

* Données de 1973.

L'examen des tableaux III et IV met en évidence une augmentation sensible de la participation du césium 137 provenant de l'ingestion des produits de la noix de coco à l'équivalent de dose efficace annuel en 1988 par rapport à 1971 à Tureia. Ceci est dû au fait qu'en 1971, l'eau de boisson, qui, sur atoll, est de l'eau de pluie recueillie en citernes, apportait une fraction non négligeable de la participation du césium 137 à la dose. Ce n'est plus le cas en 1988. 
Chez l'enfant de 5 ans, la part de l'équivalent de dose annuel dû au césium 137 des noix de coco est moins importante que celle de l'adulte, du fait d'une consommation moindre (par exemple 100 fois moins à Tureia). Par ailleurs, on remarque que l'équivalent de dose efficace annuel le plus élevé dû au césium 137 présent dans les produits de la noix de coco, celui correspondant à Tureia en 1971, est égal à $0,7 \%$ de la limite annuelle pour les populations (5000 $\mu \mathrm{Sv}^{\mathrm{an}} \mathrm{n}^{-1}$ ).

D'une façon plus directement accessible, l'interprétation sanitaire peut se faire aussi en évaluant la quantité d'eau de coco et le poids de coprah qu'il faudrait ingérer pour atteindre la dose population de $5000 \mu$ Sv.an $^{-1}$. Le tableau $V$ donne ces quantités pour les deux mêmes années de référence, avec, à titre de comparaison, les données correspondant à l'atoll de Bikini en 1987 [5].

TABLEAU $V$

Consommation journalière (exprimée en $\mathrm{kg}$ ) nécessaire pour atteindre la dose population

\begin{tabular}{|c|c|c|c|c|c|c|c|}
\hline & & Mururoa & Turela & Hao & Papeete & \multicolumn{2}{|c|}{$\begin{array}{l}\text { Atoll de Bikdn } \\
\text { Enew island Biktinl island }\end{array}$} \\
\hline \multirow{2}{*}{$\begin{array}{c}\text { Eau } \\
\text { de } \\
\text { coco }\end{array}$} & 1971 & 303,6 & 689,2 & 1184,7 & $1106,9^{*}$ & \multirow{2}{*}{$2,5^{\star \star}$} & \multirow{2}{*}{$0,2^{\star \star}$} \\
\hline & 1988 & 1191,0 & & & 1739,5 & & \\
\hline \multirow{2}{*}{ Coprah } & 1971 & 164,6 & 62,2 & 190,2 & $1404,9^{*}$ & \multirow{2}{*}{$1,2^{\star \star}$} & \multirow{2}{*}{$0,1^{\star \star}$} \\
\hline & 1988 & - 320,0 & 151,9 & 1209,6 & 1141,0 & & \\
\hline
\end{tabular}

* Données de 1973.

** Données de 1987.

On peut constater qu'à aucun moment et en aucun lieu, aucun individu n'a risqué d'atteindre la dose population avec le césium 137 par la consommation des produits de la noix de coco. Nous rappelons que la dose population considérée ici correspond à la limite annuelle de l'équivalent de dose recommandée par la CIPR pour le public. Cette limite, d'après la CIPR, garantit que le niveau de risque couramment accepté dans la vie quotidienne ne sera pas dépassé [8].

\section{CONCLUSION}

A la suite des premières expérimentations aériennes étrangères puis françaises, les retombées ont introduit dans l'environnement polynésien des radionucléides artificiels qui se sont ajoutés aux radioéléments naturels existant depuis toujours. La frange des retombées dites "locales" des essais aériens français a quelquefois concerné les atolls de Tureia et de Reao. A partir de 1975, les essais souterrains succédant aux tirs aériens, tout apport nouveau en provenance des sites d'expérimentations français a cessé en Polynésie. 
La consommation importante d'eau de coco et de coprah sur certains atolls des Tuamotu fait que la participation de ces deux aliments dans la dose efficace annuelle de la population de ces atolls est très importante, pouvant représenter plus de $75 \%$ de la dose efficace annuelle d'origine alimentaire. Mais cet apport de césium 137 par le coprah et l'eau de coco est resté très faible et ne représente, quel que soit le lieu, que quelques microsieverts par an. Ces valeurs sont négligeables par rapport à celles dues à la radioactivité naturelle en Polynésie (1000 $\mu$ sv.an ${ }^{-1}$ ).

\section{BIBLIOGRAPHIE}

[1] BABLET J.P., CAYET O. - Le monde vivant des atolls (Polynésie française, Tuamotu - Gambier). (Publication $\mathrm{n}^{\circ} 28$ de la Société des Océanistes), Paris : Musée de l'Homme, 1972.

[2] BADIE C., ARNOULD C., SARBACH J., ARNAUD M., LEMERCIER R., BERNARD C., HOWELL P., TEXIER T. - Teneur en ${ }^{90} \mathrm{Sr}$ de dents humaines collectées en Polynésie française. Radioprotection, 1987, 22, 4, 325-332.

[3] BAIR W.J., HEALY J.W., WACHHOLZ B.N. - The meaning of radiation at Bikini atoll. US Department of energy, Washington D.C., 1980.

[4] COMMUNAUTÉS EUROPÉENNES (CONSEIL) - Directive du Conseil du 3 septembre 1984, modifiant la directive 80/836/Euratom en ce qui concerne les normes de base relatives à la protection sanitaire de la population et des travailleurs contre les dangers résultant des rayonnements ionisants (84/467/Euratom). J. Off. Comm. Eur., 1984, L265 14 - (5 octobre).

[5] FINSTON R. - Radiation dosage. In : Bikini atoll rehabilitation committee, Report $\mathrm{n}^{\circ}$ 4, Status March 31, 1986, Appendix D.

[6] GROUZELLE C., DOMINIQUE M., LAFAY F., DUCOUSSO R. - Résultat d'une enquête alimentaire effectuée à Tahiti de 1980 à 1982. Rapport CEA-R-5304, $1985,108 \mathrm{p}$.

[7] GUERIN M. - Plantes utiles préeuropéennes. In : Encyclopédie de la Polynésie, vol. 2 (ch. Gleizal, Ed.). Multipress, 1986.

[8] INTERNATIONAL COMMISSION ON RADIOLOGICAL PROTECTION - Recommendation of the International commission on radiological protection (ICRP publication 26). Oxford: Pergamon Press, 1977, $53 \mathrm{p}$.

[9] MEYER J., MATHIEU J., SEGUY M., GROUZELLE C., DUCOUSSO R. - Détermination de certaines constantes chimiques d'espèces tropicales végétales et animales, destinées à l'alimentation. Rapport CEA-R-5130, 1981.

[10] MORONEY J.R. - Radioactive fallout in the southern hemisphere from nuclear weapons tests. Rapport néo-zélandais ARL-TR-013, 1979.

[11] RÉPUBLIQUE FRANÇAISE - Commissariat à l'énergie atomique et Direction des Centres d'expérimentations nucléaires. Surveillance de la radioactivité en 1988. CEA-DPS, BP 6, 92265 Fontenay-aux-Roses Cedex.

[12] UNITED NATIONS SCIENTIFIC COMMITTEE ON THE EFFECTS OF ATOMIC RADIATION (UNSCEA) - lonising radiation : sources and biological effects, report to the general assembly, New York: Nations Unies, 1982, page 221. 